Case Report

\title{
Oral Rehabilitation with Zygomatic Implants in a Patient with Cleft Palate
}

\author{
Guilherme José Pimentel Lopes de Oliveira $\left(\mathbb{D},{ }^{1}\right.$ Mariana Schaffer Brackmann, \\ Larissa Carvalho Trojan, ${ }^{3}$ Paulo Domingos Ribeiro Júnior, ${ }^{4}$ \\ and Luis Eduardo Marques Padovan (D) $^{3}$ \\ ${ }^{1}$ Department of Periodontology/Implantology, Dental School, Federal University of Uberlândia-UFU, Uberlândia 38405-266, Brazil \\ ${ }^{2}$ Department of Diagnosis and Surgery, Univ. Est. Paul.-UNESP, Araraquara 14801-903, Brazil \\ ${ }^{3}$ Instituto Latino Americano de Ensino e Pesquisa Odontológico-ILAPEO, Curitiba 80710-150, Brazil \\ ${ }^{4}$ Univ. Sagrado Coração, Bauru 17011-160, Brazil
}

Correspondence should be addressed to Luis Eduardo Marques Padovan; padovan@iocp.com.br

Received 14 February 2019; Accepted 8 April 2019; Published 6 May 2019

Academic Editor: Jamil Awad Shibli

Copyright (c) 2019 Guilherme José Pimentel Lopes de Oliveira et al. This is an open access article distributed under the Creative Commons Attribution License, which permits unrestricted use, distribution, and reproduction in any medium, provided the original work is properly cited.

\begin{abstract}
Edentulous patients with an atrophic maxilla associated with lip-palate fissures have unpredictable results after undergoing grafting procedures. In situations where the atrophic maxilla does not adequately allow reconstruction, the use of zygomatic implants has been indicated, and probably these implants can be indicated for the rehabilitation of patients with lip-palate fissures. This case report describes the oral rehabilitation treatment of a patient with a lip-palate cleft treated with zygomatic implants and implant-supported fixed prosthesis with two years of follow-up. A 65-year-old female patient had a lip-palate cleft and previously underwent surgery to close the cleft. The patient had a severely atrophic maxilla and had difficulty adapting to a removable total prosthesis. Due to the small amount of bone remaining and extensive fibrous tissue in the palate region, a rehabilitation with conventional implants associated with zygomatic implants was chosen. Two zygomatic implants and a conventional implant were placed on the right side, and a zygomatic implant and conventional implant were placed on the left side; these implants were later activated by a protocol-type prosthesis. The zygomatic implants provided an adequate aesthetic and functional outcome of the prosthesis in a patient with cleft palate.
\end{abstract}

\section{Introduction}

The use of implant-supported prostheses has been shown to be a predictable treatment with a high success rate for the treatment of any type of edentulism $[1,2]$. However, in some clinical situations, it is not possible to place implants in a good position due to the limited availability of the bone tissue $[3,4]$. In these situations, the rehabilitation of the atrophic maxilla may represent the greatest challenge because it is a bone with low biological quality compared to the mandible and contains a sinus cavity that tends to increase its degree of pneumatization after tooth loss $[3,5]$.

To address this problem, bone substitute biomaterials associated with maxillary sinus floor elevation techniques
[6], guided bone regeneration [5], osteogenic distraction [4], and the use of block grafts [7] have previously been proposed to treat the atrophic maxilla before implant placement. However, in some cases, maxillary atrophy may be of a high degree of severity that renders bone reconstruction procedures unpredictable.

Edentulous patients with an atrophic maxilla associated with lip-palate fissures have unpredictable results after undergoing grafting because they have a compromised local vascularization, low tissue elasticity, and impaired psychological aspects. In addition, the limitation of the openness of the mouth makes it difficult to place implants correctly [8]. In situations where the atrophic maxilla does not adequately allow reconstruction, the use of zygomatic implants 
has been indicated. These implants were originally designed for the treatment of maxillectomized patients who were tumour or trauma victims $[9,10]$ and may be an excellent alternative in the rehabilitative treatment of patients with lip-palate fissures [11].

This case report is aimed at describing and presenting the outcomes of the oral rehabilitation treatment of a patient with a lip-palate cleft who was treated with zygomatic implants and implant-supported fixed prosthesis after two years of follow-up.

\section{Case Presentation}

A 65-year-old female patient who presented with a lip-palate cleft previously underwent a surgical procedure to close the lip-palate cleft. However, even after this procedure, the patient had a clearly atrophic maxilla (class $\mathrm{V}$ of Cawood and Howell) and had difficulty adapting to a total removable of prosthesis. Due to the small amount of bone remaining and extensive fibrous tissue in the palate region, rehabilitation with conventional implants associated with zygomatic implants was chosen instead of subjecting the patient to a reconstruction with large bone grafts (Figure 1(a)). For the preoperative evaluation, panoramic radiography and conebeam computed tomography of the maxilla and zygomas were requested, which confirmed the low bone availability in this case (Figures 1(b) and 1(c)). Two zygomatic implants and a conventional implant were placed in the right side, and one zygomatic implant and one conventional implant were placed in the left side under general anaesthesia and nasotracheal intubation. Zygomatic implants with the Cone Morse platform (Neodent ${ }^{\circledR}$, Curitiba, Brazil) and conventional implants with the Cone Morse platform and a hydrophilic surface (Acqua surface, Neodent ${ }^{\circledR}$, Curitiba, Brazil) were used. Of these implants, a zygomatic implant with $4.4 \times$ $52.5 \mathrm{~mm}$ was installed in the region of tooth 12 , a $4.4 \times$ $40 \mathrm{~mm}$ zygomatic implant was installed in the region of tooth 16 , a zygomatic implant with $4.4 \times 52.5 \mathrm{~mm}$ was installed in the region of tooth 26 , and two conventional conical implants with $3.5 \times 11.5 \mathrm{~mm}$ were placed in the region of teeth 14 and 23. Furthermore, more than $60 \mathrm{~N} \cdot \mathrm{cm}$ of insertion torque was obtained during the placement of all the implants.

During the milling of the surgical site, the following sequence of drills was used: spherical drill zygomatic plus 2, spiral drill 2.7 zygomatic plus, pilot spiral drill zygomatic plus 2.7/3.3, spiral drill zygomatic plus 3.3, pilot spiral drill zygomatic plus 3.3/3.7, and countersink drill for Cone Morse zygomatic implant placement, and then the implant installation was performed. A surgical guide was used during the implant surgical site preparation. An antibiotic (amoxicillin) was given intraoperatively and maintained for seven days. Miniabutments were selected and installed at the time of surgery. After 24 hours, the castings were performed, and 48 hours after surgery, a screwed fixed-implant protocol prosthesis with CrCo infrastructure and acrylic teeth was installed on the implants. During the postoperative period, radiographic and clinical examinations showed that there were no complications (Figures 2(a) and 2(b)). The patient underwent follow-up after 15,30 , and 90 days and then every six months thereafter. Currently, the patient has been followed up for two years without any complaint and with a functional prosthesis (Figures 3(a)-3(c)). Furthermore, the patient expressed satisfaction with the obtained aesthetic and functional outcome. The patient gave the researchers a signed consent for the publication of this case.

\section{Discussion}

In general, it was verified in this case report that the use of zygomatic implants was effective for providing a basis for the rehabilitative treatment of a patient who presented with a lip-palate cleft. As the patient had already undergone grafting procedures and because she was not of ideal age to undergo other grafting procedures to reduce the fibrous tissue present on the palate, the grafting technique was contraindicated for the use of zygomatic implants.

Lip-palate clefts are the most frequent craniofacial malformations. These deformities were considered by the World Health Organization to be a public health problem. In 2002, this type of malformation accounted for 1 in 650 babies born worldwide [12]. The fissures present a multifactorial aetiology, with the involvement of genetic and environmental factors. Treatment of patients affected by these malformations should begin soon after birth and extend into adulthood [13]. As the patient of the present case report did not undergo treatment in the early stage, there was a reduced chance of success with the grafting procedures.

Zygomatic implants were initially designed to be used to treat atrophic maxilla to avoid graft procedures; these implants reduce the degree of morbidity of the surgical procedure and costs and accelerate prosthetic rehabilitative treatment [14]. However, it has been reported that the success of installing zygomatic implants is related to the degree of experience and skill of the surgeon and that some complications, such as haemorrhages during the procedure or the occurrence of sinusitis after the procedure, are not uncommon [15]. On the other hand, some studies have shown that zygomatic implants have high success and survival rates [10, $14,16]$, which shows that rehabilitation treatment with this type of implant is safe, as confirmed in the case report described. In this case report, the indication of the use of the zygomatic implant was due to the lacking of enough bone tissue which enables the placement of an adequate number of implants of conventional size suitable for the installation of a fixed-implant protocol prosthesis due to atrophy of the maxilla associated with the presence of the cleft palate.

It has been a tendency in medical areas to indicate less invasive surgical procedures that are associated with lower morbidity for patients $[17,18]$. Less invasive procedures accelerate the healing process and generate a high degree of satisfaction of patients, and whenever possible, less invasive surgical procedures should be indicated. Although grafting procedures are related to high rates of clinical success, this additional surgical step requires more time due to implant placement in most cases $[14,16]$. In addition, in cases of severe atrophy, the use of an autogenous bone graft isolated or associated with other bone substitutes is more appropriate because it is necessary that the graft presents good biological 


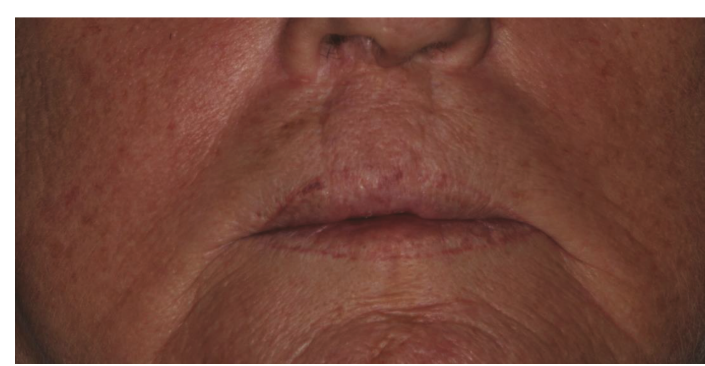

(a)

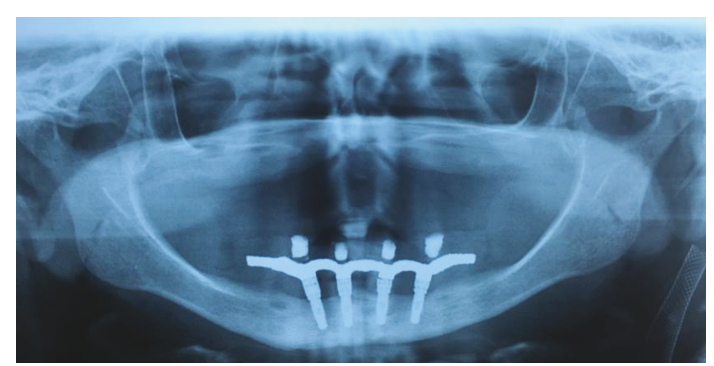

(b)

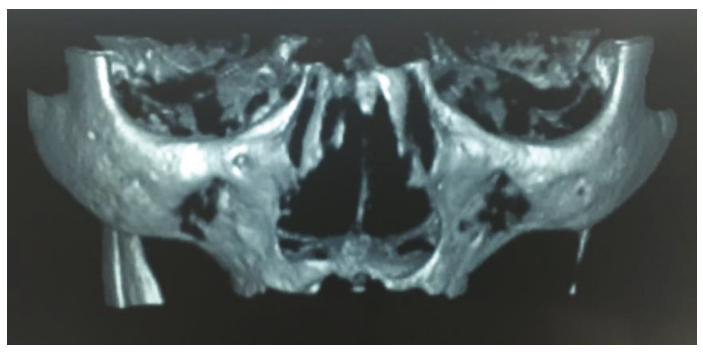

(c)

FIGURE 1: (a) Preoperative clinical condition of the patient, (b) preoperative panoramic radiography, and (c) preoperative cone beam computed tomography. Note the large degree of maxilla atrophy presented by the patient before the surgical procedure.

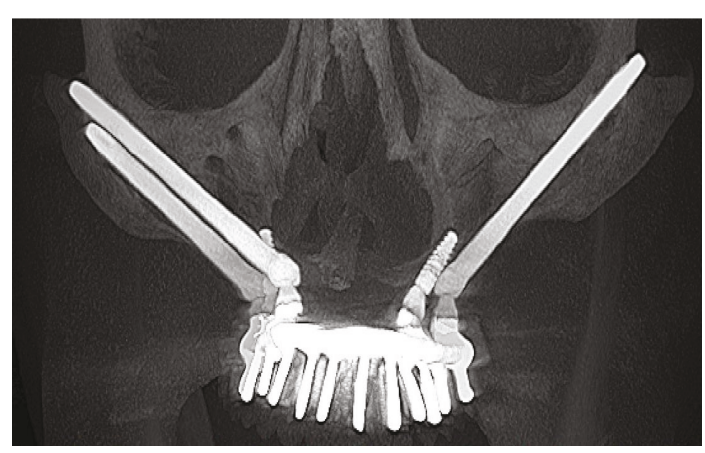

(a)

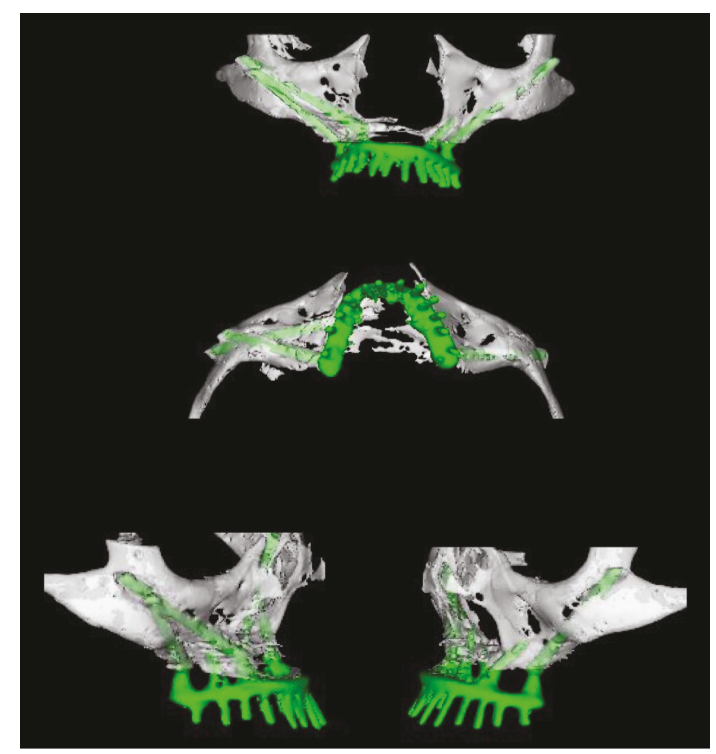

(b)

Figure 2: (a) Panoramic tomography and (b) tomographic image of the patient after 2 years of follow-up. Note the good position of the implants placed and the absence of complications.

properties to integrate into the surgical beds of the native bone with little thickness [5]. In these cases, morbidity in the donor sites generates a great discomfort to patients [19]. In fact, the option to install zygomatic implants in this case promoted the application of a more conservative surgical technique with a smaller possibility of causing morbidity to the patient and promoted acceleration of prosthetic rehabilitation, thus promoting a greater patient satisfaction with the surgical procedure performed.
Another possibility for rehabilitation of this case would be the use of overdenture-type prosthesis that has been shown to be a conservative treatment with high rates of success and patient satisfaction [20]. However, in this specific case, the patient's previous positive experience with the protocol-fixed prostheses used to rehabilitate the inferior jaw was taken into account. In addition, there was no communication of the nasal with the buccal cavity due to the grafting attempt that sealed this communication. 


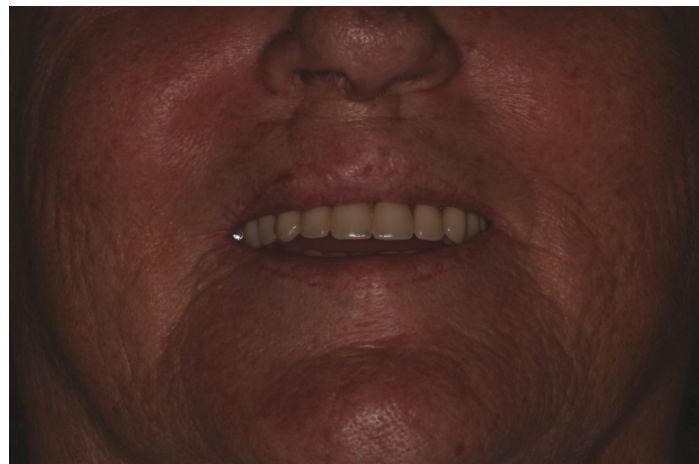

(a)

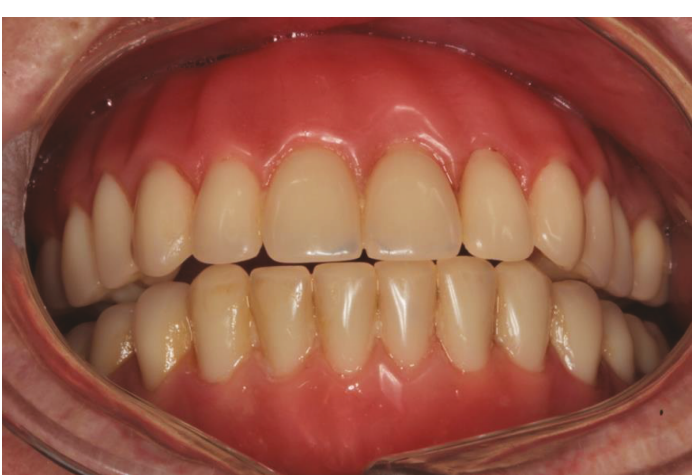

(b)

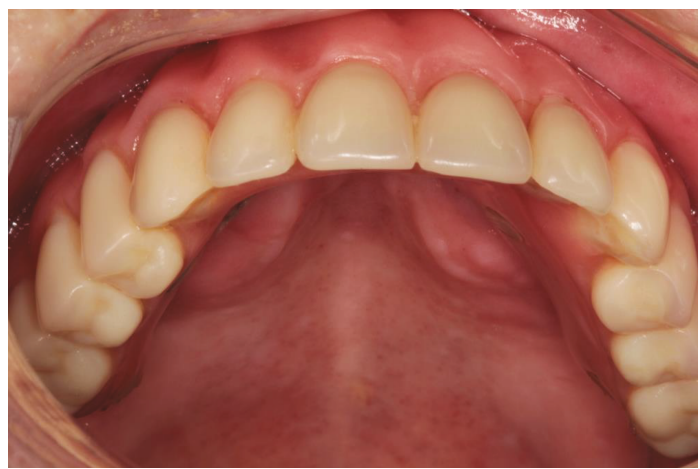

(c)

Figure 3: (a) Postoperative clinical condition of the patient with the upper protocol-type prosthesis in function, (b) postoperative condition of the prosthesis, and (c) postoperative condition of the palate. Note the good clinical outcome of the upper protocol-type prosthesis after 2 years of follow-up. Furthermore, a good condition of the palate with the communication of the nasal and oral cavities is showed.

\section{Conclusion}

This case report showed that the use of zygomatic implants in a severely atrophic maxilla provides a sufficiently stable support for the installation of a total-fixed prosthesis in the atrophic maxilla of a lip-palate cleft patient with good clinical outcomes after two years of follow-up.

\section{Conflicts of Interest}

The authors have no conflicts of interest to declare.

\section{References}

[1] T. J. Balshi, G. J. Wolfinger, R. W. Slauch, and S. F. Balshi, "A retrospective analysis of 800 Brånemark System implants following the All-on-Four ${ }^{\mathrm{TM}}$ protocol," Journal of Prosthodontics, vol. 23, no. 2, pp. 83-88, 2014.

[2] P. Maló, M. de Araújo Nobre, A. Lopes, A. Ferro, and I. Gravito, "Single-tooth rehabilitations supported by dental implants used in an immediate-provisionalization protocol: report on long-term outcome with retrospective follow-up," Clinical Implant Dentistry and Related Research, vol. 17, Supplement 2, pp. e511-e519, 2015.

[3] C. Aparicio, W. Ouazzani, R. Garcia, X. Arevalo, R. Muela, and V. Fortes, "A prospective clinical study on titanium implants in the zygomatic arch for prosthetic rehabilitation of the atrophic edentulous maxilla with a follow-up of 6 months to 5 years," Clinical Implant Dentistry and Related Research, vol. 8, no. 3, pp. 114-122, 2006.

[4] T. Binger, M. Rücker, and W. J. Spitzer, "Dentofacial rehabilitation by osteodistraction, augmentation and implantation despite osteogenesis imperfecta," International Journal of Oral \& Maxillofacial Surgery, vol. 35, no. 6, pp. 559-562, 2006.

[5] I. A. Urban, A. Monje, J. L. Lozada, and H. L. Wang, "Longterm evaluation of peri-implant bone level after reconstruction of severely atrophic edentulous maxilla via vertical and horizontal guided bone regeneration in combination with sinus augmentation: a case series with 1 to 15 years of loading," Clinical Implant Dentistry and Related Research, vol. 19, no. 1, pp. 46-55, 2017.

[6] A. Wildburger, M. Payer, N. Jakse, D. Strunk, N. EtchardLiechtenstein, and S. Sauerbier, "Impact of autogenous concentrated bone marrow aspirate on bone regeneration after sinus floor augmentation with a bovine bone substitute-a split-mouth pilot study," Clinical Oral Implants Research, vol. 25, no. 10, pp. 1175-1181, 2014.

[7] B. A. Gultekin, E. Cansiz, and M. O. Borahan, "Clinical and 3dimensional radiographic evaluation of autogenous iliac block bone grafting and guided bone regeneration in patients with atrophic maxilla," Journal of Oral and Maxillofacial Surgery, vol. 75, no. 4, pp. 709-722, 2017.

[8] K. Wermker, S. Jung, U. Joos, and J. Kleinheinz, "Dental implants in cleft lip, alveolus, and palate patients: a systematic review," The International Journal of Oral \& Maxillofacial Implants, vol. 29, no. 2, pp. 384-390, 2014. 
[9] A. V. Pham, M. Abarca, A. de Mey, and C. Malevez, "Rehabilitation of a patient with cleft lip and palate with an extremely edentulous atrophied posterior maxilla using zygomatic implants: case report," The Cleft Palate-Craniofacial Journal, vol. 41, no. 5, pp. 571-574, 2017.

[10] E. M. Sartori, L. E. M. Padovan, I. A. de Mattias Sartori, P. D. Ribeiro Jr, A. C. Gomes de Souza Carvalho, and M. C. Goiato, "Evaluation of satisfaction of patients rehabilitated with zygomatic fixtures," Journal of Oral and Maxillofacial Surgery, vol. 70, no. 2, pp. 314-319, 2012.

[11] C. Aparicio, C. Manresa, K. Francisco et al., "The long-term use of zygomatic implants: a 10-year clinical and radiographic report," Clinical Implant Dentistry and Related Research, vol. 16, no. 3, pp. 447-459, 2014.

[12] W. Shaw, "Global strategies to reduce the health care burden of craniofacial anomalies: report of WHO meetings on international collaborative research on craniofacial anomalies," The Cleft Palate-Craniofacial Journal, vol. 41, no. 3, pp. 238-243, 2004.

[13] W. A. Pena, K. Vargervik, A. Sharma, and S. Oberoi, "The role of endosseous implants in the management of alveolar clefts," Pediatric Dentistry, vol. 31, no. 4, pp. 329-333, 2009.

[14] R. Davó, P. Felice, R. Pistilli et al., "Immediately loaded zygomatic implants vs conventional dental implants in augmented atrophic maxillae: 1-year post-loading results from a multicentre randomised controlled trial," European Journal of Oral Implantology, vol. 11, no. 2, pp. 145-161, 2018.

[15] B. R. Chrcanovic and M. H. N. G. Abreu, "Survival and complications of zygomatic implants: a systematic review," Oral and Maxillofacial Surgery, vol. 17, no. 2, pp. 81-93, 2013.

[16] M. Esposito, R. Davó, C. Marti-Pages et al., "Immediately loaded zygomatic implants vs conventional dental implants in augmented atrophic maxillae: 4 months post-loading results from a multicentre randomised controlled trial," European Journal of Oral Implantology, vol. 11, no. 1, pp. 11-28, 2018.

[17] M. Motta, R. Monsano, G. R. Velloso et al., "Guided surgery in esthetic region," Journal of Craniofacial Surgery, vol. 27, no. 3, pp. e262-e265, 2016.

[18] Y. N. R. Gallardo, I. R. T. da Silva-Olivio, E. Mukai, S. Morimoto, N. Sesma, and L. Cordaro, "Accuracy comparison of guided surgery for dental implants according to the tissue of support: a systematic review and meta-analysis," Clinical Oral Implants Research, vol. 28, no. 5, pp. 602-612, 2017.

[19] E. Nkenke and F. W. Neukam, "Autogenous bone harvesting and grafting in advanced jaw resorption: morbidity, resorption and implant survival," European Journal of Oral Implantology, vol. 7, Supplement 2, pp. S203-S217, 2014.

[20] M. Sánchez-Siles, J. F. Ballester-Ferrandis, N. Salazar-Sánchez, F. J. Gómez-García, R. Moraleja-Ruiz, and F. CamachoAlonso, "Long-term evaluation of quality of life and satisfaction between implant bar overdentures and conventional complete dentures: a 23 years retrospective study," Clinical Implant Dentistry and Related Research, vol. 20, no. 2, pp. 208-214, 2018. 


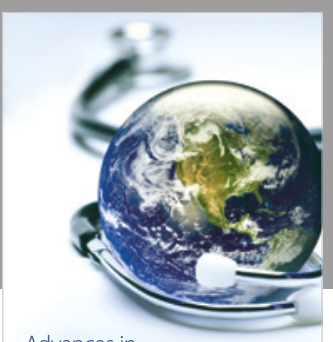

Advances in
Public Health

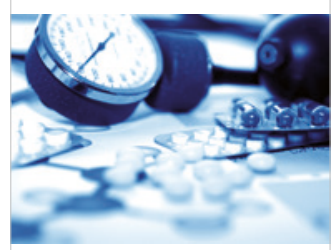

Case Reports in

Medicine

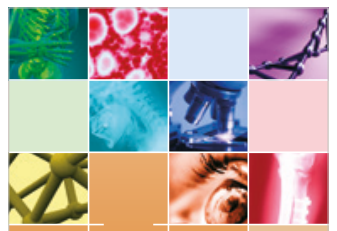

niernational Journal of

Biomaterials
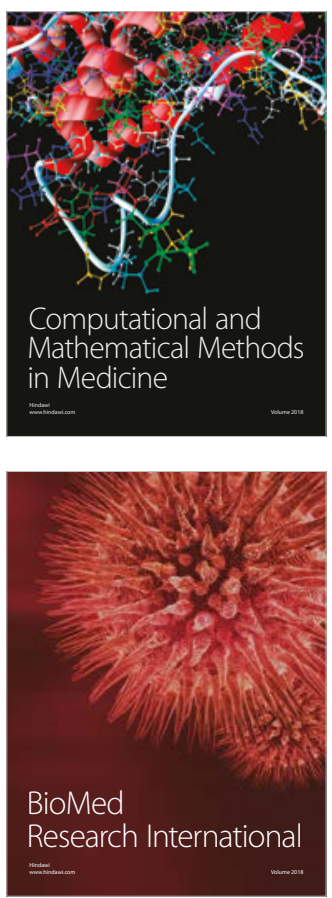

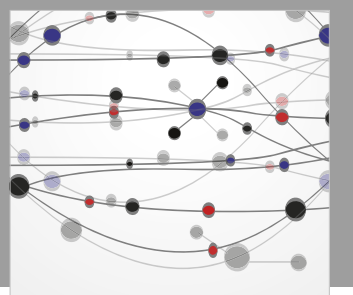

The Scientific World Journal Dentistry

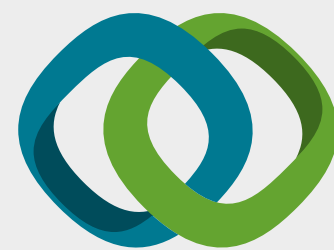

Hindawi

Submit your manuscripts at

www.hindawi.com
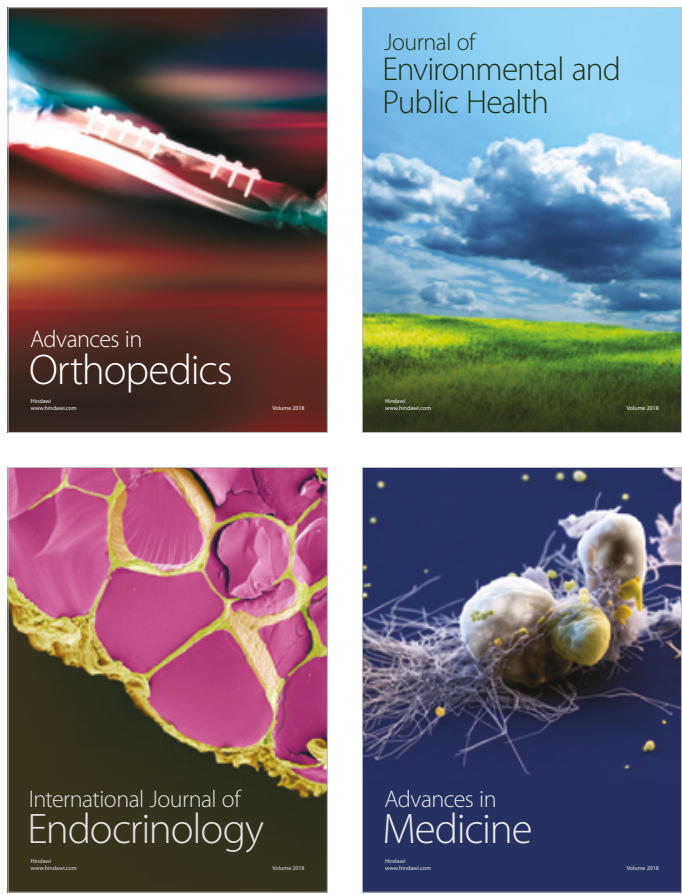
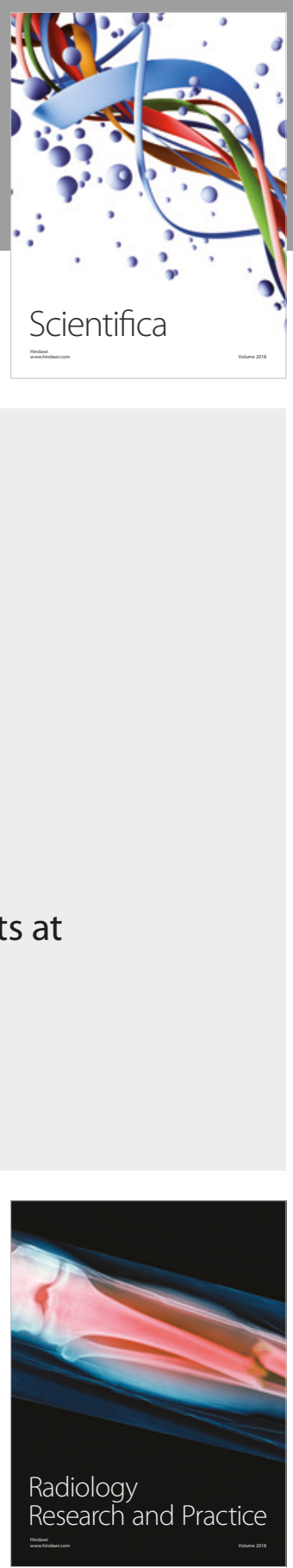

Scientifica

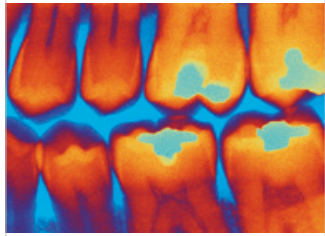

Case Reports in

Dentistry
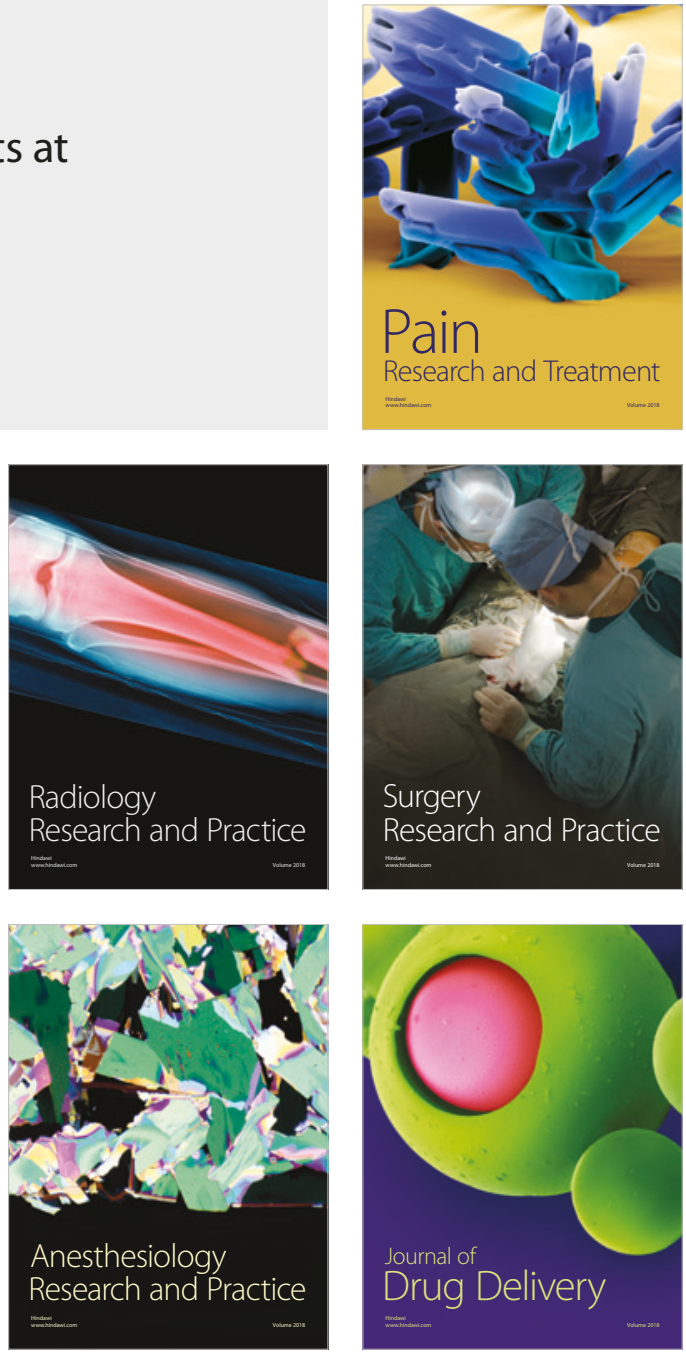